\title{
Comments on A new class of nonlinear conjugate gradient coefficients with global convergence properties *
}

\author{
Zhifeng Dai ${ }^{\dagger}$ \\ College of Mathematics and Computational Science, Changsha University of \\ Science and Technology, Changsha 410114, China
}

\begin{abstract}
In Rivaie et al [M. Rivaie, M. Mustafa, L.W. June and I. Mohd, A new class of nonlinear conjugate gradient coefficient with global convergence properties, Appl. Math. Comp, 218(2012), 11323-11332], an efficient CG algorithm has been proposed for solving unconstrained optimization problems. However, due to a wrong inequality (3.3) used in Rivaie et al., the proof of theorem 2 and the global convergence theorem 3 are not correct. We present the necessary corrections, then the proposed method in Rivaie et al still converges globally. Finally, we report some numerical comparisons.

Keywords: Unconstrained optimization, Conjugate gradient method, Global convergence.
\end{abstract}

Mathematics Subject Classification (2000) 90C30, 65K05.

\section{Introduction}

Due to low memory requirements and strong global convergence property, nonlinear conjugate gradient methods are efficient for solving the following unconstrained optimization problem,

$$
\min f(x), \quad x \in R^{n}
$$

where $f: R^{n} \rightarrow R$ is a continuously differentiable function, especially if the dimension $n$ is large.

The iterates of conjugate gradient methods for solving (1.1) are obtained by

$$
x_{k+1}=x_{k}+\alpha_{k} d_{k} .
$$

where $\alpha_{k}$ is a steplength. The steplength $\alpha_{k}$ is computed by carrying out some line search, and $d_{k}$ is the search direction defined by

$$
d_{k}=\left\{\begin{array}{lll}
-g_{k}, & \text { if } \quad k=1, \\
-g_{k}+\beta_{k} d_{k-1}, & \text { if } \quad k \geq 2,
\end{array}\right.
$$

${ }^{*}$ This work is supported by the NSF of China granted 11301041, Project funded by China Postdoctoral Science Foundation(2015T80893), Natural Science Foundation of Hunan Province(2015JJ3015).

${ }^{\dagger}$ E-mail address: zhifengdai823@163.com.

(C) 2016. This manuscript version is made available under the Elsevier user license http://www.elsevier.com/open-access/userlicense/1.0/ 
where $\beta_{k}$ is a scalar, $g_{k}$ is gradient of $f(x)$ at $x_{k}$. Varieties of this method differ in the way of selecting $\beta_{k}$.

In the recent paper[1, Rivaie et al. proposed a new class of nonlinear conjugate gradient coefficients which is called RMIL method. The parameter $\beta_{k}$ in RMIL method is computed as follows

$$
\beta_{k}^{\mathrm{RMIL}}=\frac{g_{k}^{T}\left(g_{k}-g_{k-1}\right)}{\left\|d_{k-1}\right\|^{2}}=\frac{g_{k}^{T}\left(g_{k}-g_{k-1}\right)}{\left\|d_{k-1}\right\|^{2}} .
$$

By using the following exact line search,

$$
f\left(x_{k}+\alpha_{k} d_{k}\right)=\min _{\alpha \geq 0} f\left(x_{k}+\alpha d_{k}\right),
$$

the search direction $d_{k}$ satisfies the sufficient descent condition:

$$
g_{k}^{T} d_{k}=-\left\|g_{k}\right\|^{2}, \quad \forall k \geq 0 .
$$

Numerical comparisons show that this computational scheme outperforms some other conjugate gradient methods. However, due to a wrong inequality (3.3) used in Rivaie et al.[1], the proof of theorem 2 and the global convergence theorem 3 is not correct. In what follows, the necessary corrections will be presented.

\section{Comments on the convergence of RMIL algorithm}

Here, we firstly point out a wrong inequality used in Rivaie et al.[1], namely inequality (3.3) about $\beta_{k}^{\mathrm{RMIL}}$, which plays a key role in global convergence analysis of Theorem 2 and Theorem 3.

In order to make the convergence proof easier, Rivaie et al. [1] first provided an upper bound for the coefficient $\beta_{k}^{R M I L}$. At (3.2) in [1], Rivaie et al. defined

$$
\beta_{k+1}^{\mathrm{RMIL}}=\frac{g_{k+1}^{T}\left(g_{k+1}-g_{k}\right)}{\left\|d_{k}\right\|^{2}}=\frac{\left\|g_{k+1}\right\|^{2}-g_{k+1}^{T} g_{k}}{\left\|d_{k}\right\|^{2}},
$$

and stated that

$$
0 \leq \beta_{k+1}^{\mathrm{RMIL}} \leq \frac{\left\|g_{k+1}\right\|^{2}}{\left\|d_{k}\right\|^{2}}
$$

Since the sign of $g_{k+1}^{T} g_{k}$ in 2.1 cannot be identified as positive or negative, we can not obtain $2.2)((3.3)$ in [1]). However, in the proof of Theorem 2 and Theorem 3 in [1, the inequality (2.2) ((3.3) in [1]) plays a critical role in global convergence analysis.

In order to accomplish the global convergence analysis of the RMIL method, we present a RMIL+ coefficient as follows

$$
\beta_{k+1}^{\mathrm{RMIL}+}=\left\{\begin{array}{lll}
\frac{g_{k+1}^{T}\left(g_{k+1}-g_{k}\right)}{\left\|d_{k}\right\|^{2}}, & \text { if } \quad 0 \leq g_{k+1}^{T} g_{k} \leq\left\|g_{k+1}\right\|^{2}, \\
0, & \text { otherwise }
\end{array}\right.
$$

It is obvious that $\beta_{k+1}^{\mathrm{RMIL}+}$ satisfies $[2.2)((3.3)$ in [1] $)$.

Following the same proof as Theorem 2 and Theorem 3 in [1], we can get global convergence with exact line searches. 


\section{Numerical experiments}

This section reports some numerical experiments to compare the efficiency of RMIL method in [1] and RMIL+ method. We use the same test problems with the same initial points in [1].

The tested methods have the following meanings:

- "RMIL" stands for the RMIL method with the exact line search in [1];

- "RMIL+" stands for the RMIL+ method (2.3) with the exact line search.

The stop criterion is as follows: the program is stopped if the inequality $\left\|g_{k}\right\|_{\infty} \leq 1.0 E-6$ is satisfied. All codes were written in MatlabR2013a and run on PC with 2.10GHz CPU processor, 2.0GB RAM memory, and Windows 2007 operating system.

Numerical results are compared based on the number of iterations and CPU time. In some cases, the computation stopped due to the failure of a line search to find the positive stepsize, and thus it was considered a failure.

The performance results of the CPU time and number of iterations are shown in Figures. 1 and 2, respectively, using a performance profile introduced by Dolan and More [2]. For example, in the figure 1, the performance profiles by Dolan and More to compare the performance based on the CPU time between the RMIL method in [1] and RMIL+ method. That is, for each method, we plot the fraction $\mathrm{P}$ of problems for which the method is within a factor $\tau$ of the best time. The left side of the figure gives the percentage of the test problems for which a method is the fastest; the right side gives the percentage of the test problems that are successfully solved by each of the

methods. The top curve is the method that solved the most problems in a time that is within a factor $\tau$ of the best time.

For the given test function in [1], both RMIL+ method and RMIL method can solve all of the test problems. Moreover, the initial points do not influence the result. These show that the RMIL+ method and RMIL method have good performance for the given test function in [1]. Comparing the CPU time of the RMIL+ method with the RMIL method, we find that the RMIL+ method is slightly better than the RMIL method, and the differences are not significant. Assessing the number of iterations within the tested methods, we see that the RMIL+ method and RMIL method enjoy almost the same performance.

From figures 1 and 2, we can see numerical performance for the RMIL+ method and RMIL method is almost the same. The main contribution of this note is to accomplish global convergence analysis of the RMIL method in [1].

\section{References}

[1] M. Rivaie, M. Mustafa, L.W. June and I. Mohd, A new class of nonlinear conjugate gradient coefficient with global convergence properties, Appl. Math. Comp, 218(2012),11323-11332. 
[2] E. Dolan, J.J. More, Benchmarking optimization software with performance profile, Math. Prog. 91 (2002) $201-213$.

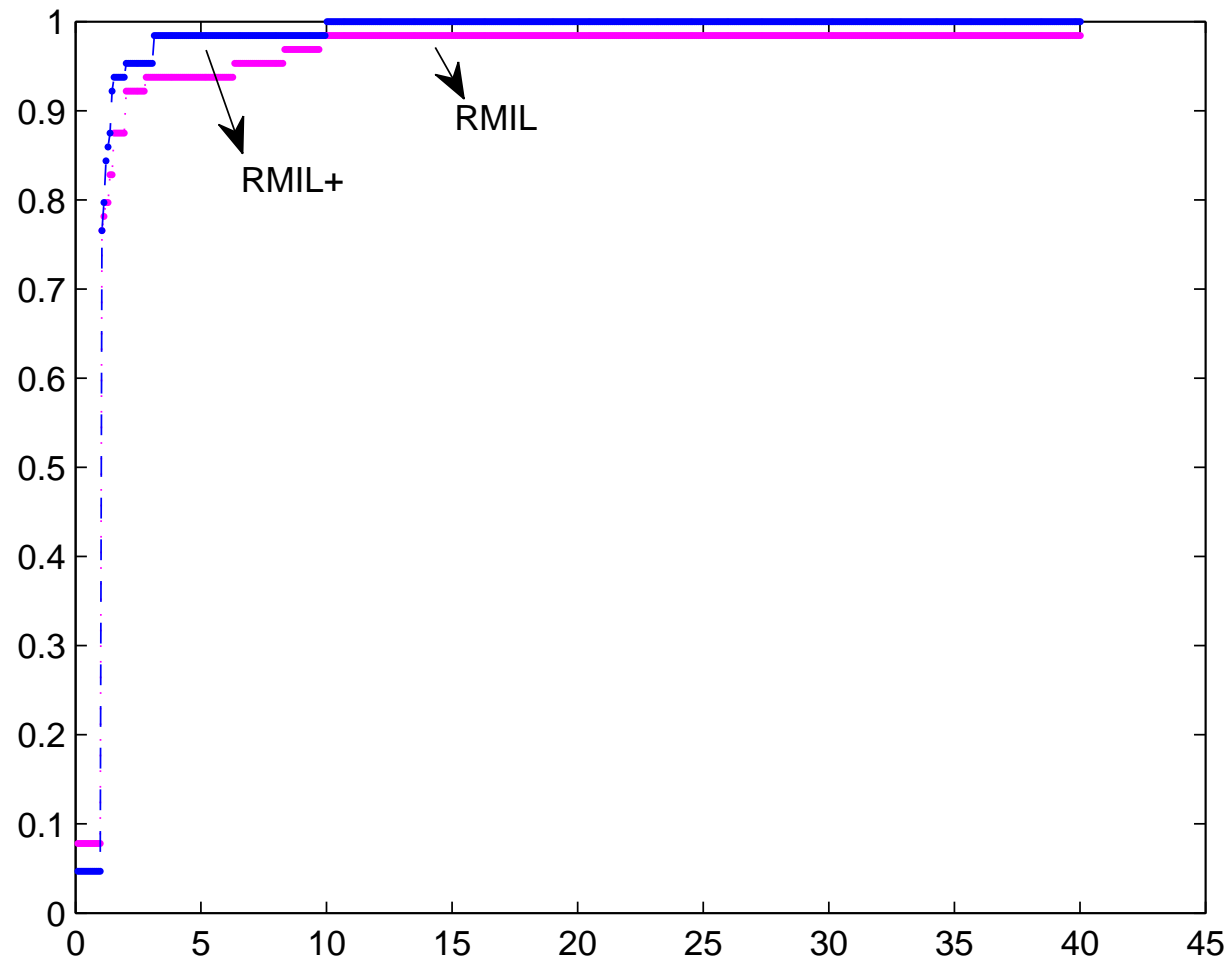

Figure 1-Performance profiles with respect to CPU time. 


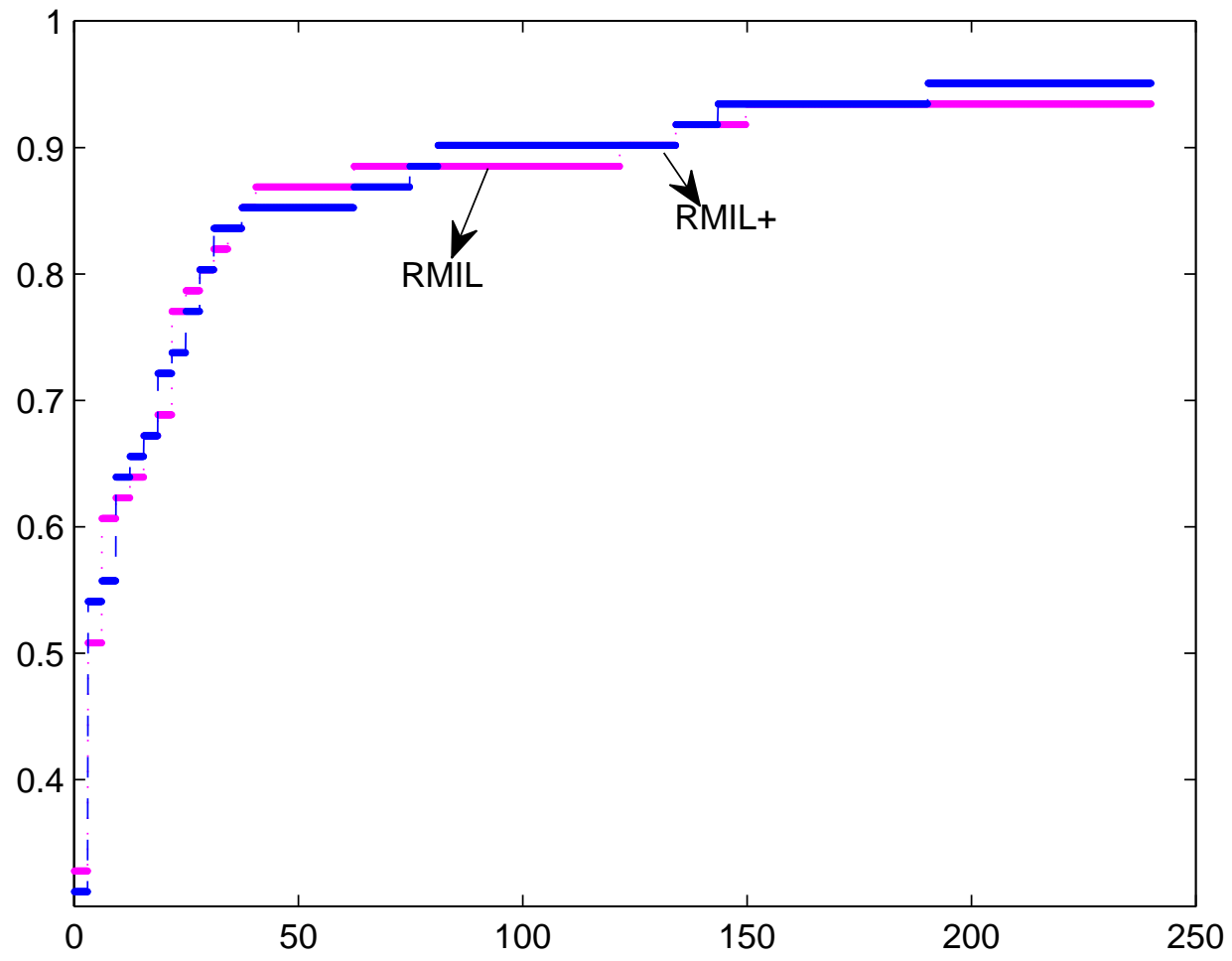

Figure 2-Performance profiles with respect to the number of iterations. 